\title{
Manual-based psychotherapies in clinical practice Part 1: assets, liabilities, and obstacles to dissemination
}

\begin{abstract}
Treatment manuals evolved in comparative psychotherapy outcome studies but are rapidly becoming a major medium for disseminating empirically supported treatments. Currently, the role of manual-based psychotherapies in clinical practice is a source of considerable debate among mental health practitioners and clinical researchers. In this editorial, we address why translating manual-based treatments from research to practice may be both difficult and worthwhile. We begin with an outline of the arguments for and against manuals and then explore some of the concrete difficulties involved in translating manuals into practice. Finally, we briefly address how these difficulties might be handled.

Perhaps the most often articulated criticism of manual-based treatments is that they lead to a bland, rule governed, and emotionally detached form of therapy. Some therapists fear that practising therapy under these conditions may feel more like following a recipe than the intuitive and creative process that drew therapists to the field. Another criticism leveled against manual-based treatments is that clinical situations rarely mirror the tightly controlled conditions of a clinical research study. ${ }^{1}$ Criteria for participation in psychotherapy research often require accurate and differential diagnosis, which can be time consuming and costly. In addition, people with multiple diagnoses (eg, panic, depression, and substance dependence) are often deemed ineligible for participation in research. Yet another criticism lies in the impracticality of having a separate treatment manual for every diagnosis. A separate treatment manual for each diagnosis makes acquiring training and achieving competence in empirically supported treatments all but impossible because there is simply too much to learn. This trend toward differential treatment based on diagnosis is puzzling since few data support the use of alternative forms of psychotherapy based on diagnosis. ${ }^{2}$
\end{abstract}

\footnotetext{
Arguments in favour of manual-based treatments

Although some psychologists oppose manual-based treatments, others strongly favour them. The arguments in favour of manual-based treatment rest on its ability to capitalise on an actuarial approach to clinical decision making, and in so doing, avoid many of the pitfalls of clinical judgment. Manual-based treatments are also supported by empirical research and thereby ensure that clients are receiving interventions that have been shown to be effective, rather than those that are simply popular or seem helpful to practising clinicians. In addition, the structured and time limited nature of manual-based treatments often results in more focused treatments that facilitate patients' active engagement in the treatment process. ${ }^{3}$ In a similar vein, treatment manuals make supervision easier and facilitate dissemination of psychotherapies. From the clinicians' perspective, knowledge of manualised treatments broadens the repertoire of treatment skills available and encourages greater technical eclecticism. ${ }^{4}$ Also, counterintuitive though it may
}

seem, the use and proliferation of manuals has actually focused attention on patients who do not respond to treatment. ${ }^{4}$ Finally, Addis argues that knowing a treatment is empirically supported can help clinicians to feel confident and optimistic when working with particularly difficult clients. ${ }^{5}$ This point can be a boon to any therapist, as clinical work, while often rewarding, can also be draining and disheartening.

\section{Barriers to translating manual-based treatments into practice}

Despite the positive points listed above, some concrete barriers make it difficult to translate empirically supported treatments from research into practice. At the most basic level, using manual-based therapies is difficult because so few clinicians are trained to provide manual-based treatments, or even know what they are. Addis and Krasnow surveyed a national sample of licensed psychologists in practice. ${ }^{6}$ They found that $77 \%$ of therapists had heard of treatment manuals, leaving a full $23 \%$ who had not. They also found that $37 \%$ of those surveyed reported having a "somewhat" to "totally" unclear idea of what a treatment manual is. Furthermore, they found that of the practising psychologists they surveyed, $47 \%$ reported never having used a treatment manual. These findings suggest that psychologists as a group have had limited experience with treatment manuals. In addition, Addis and Krasnow found that therapists' understandings of what manuals were informed their attitudes about them; those who viewed manuals as rigid guides to treatment that impeded creativity and the therapeutic alliance viewed manuals negatively, whereas those who had more flexible views of manuals regarded them more positively. ${ }^{6}$ Thus, if dialogue about this topic is to proceed in a constructive fashion, education about what manuals are and how they can and should be used is imperative.

Exactly how practitioners might glean knowledge about manual-based treatments is a complicated issue. Not all graduate programmes include training in manual-based treatments as part of their curriculum. As a result, many students graduate from doctoral programmes in psychology without ever having practised a manual-based treatment. Furthermore, many graduate students experience conflicting feelings about research and practice. ${ }^{7}$ Although they conduct and consume research in graduate school, their actual clinical work in therapy often seems far removed from the world of research. As a result, clinical students sometimes feel as though research and clinical practice are disparate. One solution to this problem would entail graduate programmes changing their curricula to include training in manual-based treatments. In this way, research and practice would be more obviously connected, and students would complete their training with a clear idea of what an empirically supported treatment is. In addition, internship training is an ideal context in which to explore some of the more complex issues that arise in using manual-based treatments. ${ }^{8}$ In 
the meantime, however, most clinicians will have to learn about manual-based treatments outside of academia.

Currently, most clinicians receive training in manual-based treatments after they have completed their training and become professionals, if they receive any such training at all. Although licensed clinicians often attend workshops to earn continuing education credits, most workshops are too short to provide the time and supervision necessary to master a manual-based treatment. Most clinicians are already overextended and would have difficulty finding the time, energy, or money to pursue extensive training in empirically supported treatments. Pursuing such training would often entail taking time off from paid work. In addition, the common 6 session limit of managed care is insufficient to complete most manualised treatments, which usually require between 12 and 20 sessions.

Another concrete obstacle to translating manual-based treatments into practice is that once clinicians have received training, many treatment developers believe additional supervision is necessary to achieve a high level of skill in a treatment. Few mental health agencies have the time or financial resources to provide this supervision, and even if they did, the dearth of adherence and competence measures to assess treatment fidelity makes such a job difficult. The proliferation of treatment manuals for specific disorders is another problem with training clinicians in empirically supported treatments. It may be impossible, given the time constraints of treatment within a managed care system, to take the time to differentially diagnose a client. Even if a thorough diagnostic assessment process were possible, it would be difficult for most clinicians to receive training in such a wide variety of manual-based treatments.

Given the limits on the time and money available for mental health treatment in general, perhaps a different approach to providing empirically supported treatments would make disseminating them easier. Rather than learning a different treatment for each and every possible diagnosis, we might adopt a more generalist approach that could be applied across diagnoses. For example, Persons has developed a manual that allows clinicians to choose from a variety of cognitive behavioural therapy (CBT) techniques. ${ }^{9}$ This approach to using a manual-based treatment calls for flexibility and creativity, which may make it more appealing to therapists who would otherwise turn away from manual-based treatments. Although Persons' model is based on CBT, other therapeutic genres could create similar manuals. For example, behaviour therapists could develop a generalist strategy that emphasises the importance of a credible treatment rationale, monitoring cognitive processes, analyzing and understanding the contexts in which behaviours occur, and rehearsing adaptive behaviours. ${ }^{5}$ Flexibility and wide applicability may make generalist approaches like this one the most practical way of using empirically supported treatments.

\section{Conclusions}

It is clear that the obstacles in translating empirically supported treatments into practice are many. If these obstacles are to be surmounted, change must occur at several levels. Graduate training in clinical psychology must include training in manualbased treatments, and mental health agencies must provide training and supervision in empirically supported treatments. If clinicians are to have a better understanding of manual-based treatments, their exposure to them must be more extensive, and agencies that provide mental health services must address this issue by prioritizing training and providing time and compensation for it. At the bureaucratic level, managed care organisations and insurance companies must recognise the value of empirically supported treatments and change their policies to enable therapists to provide them.

In summary, while there are some legitimate concerns about manual-based treatments that require attention and thought, empirically supported treatments have much to recommend them. By capitalising on actuarial data, they encourage therapists to take greater responsibility for their work and to think critically about why they do what they do. Despite these positive points, opinions about manuals remain polarised. While we may be able to surmount some of the practical obstacles to disseminating empirically supported treatments, as a field we must address practitioner acceptance of empirically supported treatments. Perhaps most importantly, we must wrestle with the ultimate question of whether manuals should guide clinical practice. We will address these questions in part 2 of this series.

\section{ABIGAIL K MANSFIELD, MA MICHAEL E ADDIS, PhD Clark University \\ Worcester, Massachusetts, USA}

1 Fensterheim H, Raw SD. Psychotherapy research is not psychotherapy practice. Clinical Psychology: Science and Practice 1996;3:168-71.

2 Addis ME, Carpenter KM. Treatment manuals and the future of behavior therapy. The Behavior Therapist 1997;20:53-5.

3 Wilson GT. Manual-based treatment and clinical practice. Clinical Psychology: Science and Practice 1998;5:363-75.

4 Wilson GT. Manual-based treatments: the clinical application of research findings. Behav Res Ther 1996;34:295-314.

5 Addis ME. Evaluating the treatment manual as a means of disseminating empirically validated psychotherapies. Clinical Psychology: Science and Practice 1997;4:1-11.

6 Addis ME, Krasnow AD. A national survey of practicing psychologists' attitudes towards psychotherapy treatment manuals. J Consult Clin Psychol 2000;68:331-9.

7 Addis ME. Graduate training in Boulder model clinical psychology programs: the evolving tension between science and practice. In: Soldz S, McCullough L, editors. Reconciling empirical knowledge and clinical experience: the art and science of psychotherapy. Washington: APA Press, 2000;51-66.

8 Calhoun KS, Moras K, Pilkonis PA, et al. Empirically supported treatments: implications for training. J Consult Clin Psychol 1998;66:151-62.

9 Persons JB. Cognitive therapy in practice: a case formulation approach. New York: Norton, 1989. 